\section{Samuel Martin} Professor,

School of Civil Engineering Georgia Institute of Technology. Atlanta, Ga.

H. Medlarz

Graduate Research Assistant, Institute for Hydromechanics. University of Karlsruhe Karisruhe, West Germany

\section{C. Wiggert \\ Associate Professor Department of Civil Engineering. Michigan State University, \\ East Lansing, Mich.}

\section{Brennen}

Associate Professor, Department of Mechanical Engineering, California Institute of Technology,

Pasadena, Calif

\title{
Cavitation Inception in Spool Valves
}

Cavitation has been investigated in directional control valves in order to identify damage mechanisms characteristic of components of aircraft hydraulic systems. Tests have been conducted in a representative metal spool valve and in a model three times larger. Data taken under noncavitating conditions with both valves showed that the position of the high-velocity annular jet shifts orientation, depending upon valve opening and Reynolds number. By means of high-frequency response pressure transducers strategically placed in the valve chamber cavitation could be sensed by the correlation of noise with a cavitation index. The onset of cavitation can be detected by comparing energy spectra for a fixed valve opening and a constant discharge. Another sensitive indicator of cavitation inception is the ratio of cavitating to noncavitating spectral densities. The incipient cavitation number as defined in this investigation is correlated with the Reynolds number for both valves.

\section{Introduction}

Cavitation is not nearly as well documented in oil hydraulic systems as it is in such water hydraulic systems as pumps, propellers, hydraulic turbines, and hydrofoils. In aircraft hydraulic systems, cavitation most frequently occurs in system valves, pumps, and actuators. Large differences in pressure is a frequent cause of small-scale cavitation in chambers of four-way spool valves, while high frequency motion of a valve-controlled actuator can lead to large-scale cavitation in the cylinder. Another source of cavitation in aircraft hydraulic systems is the improper filling of the pistons on an axial-piston pump. Either during transient loading or under steady-state operation, cavitation can occur in the return chamber of directional control valves because of the Iarge pressure drop across the orifice. It is of interest to know the potential cavitation damage, as well as any effect of cavitation on system performance under both steady and unsteady flow conditions. Criteria should be established for the onset of cavitation, and damage mechanisms need be identified once cavitation is extensive.

The amount of literature on cavitation in oil hydraulic components is rather sparse. The major publications in the English language are referred to in the book by McCloy and Martin [1]. There is also important literature in German, principally from the fluid power group at the Technical University of Aachen. Cavitation was investigated to a limited extent by MacLellan, et al. [2], in their laboratory study in an enlarged two-dimensional model of a piston type control

Contributed by the Fluids Engineering Division of The AMERICAN SUCIETY OF Mechanical Engtneers and presented at the International Symposium on Cavitation Inception, ASME Winter Annual Meeting. New York, N.Y., December 2-7. 1979. Manuscript received by the Fluids Enginecring Division March 18, 1980. valve. Later, but more complete, work on cavitation in spool valves was reported by McCloy and Beck [3], who established criteria for cavitation inception in two valves, both two dimensional, one with water and the other with oil as the test liquid. Backé and Benning [4], Backé and Riedel [5], and Riedel [6] all report on cavitation in short orifices using hydraulic oil. Recent work by Kleinbreuer [7, 8] has enhanced the understanding of the effect of dissolved gas, viscosity, and jet proximity on erosion of various metals by high velocity discharge of hydraulic oil from an orifice. Eich $[9,10]$ applied the diagnostic tool of noise detection in his investigation of cavitation in oil hydraulic components. The effect of gas content and orifice length on desinent and incipient cavitation is reported by Lichtarowicz and Pearce [11]. Flow pattern changes under both noncavitating and cavitating flow conditions were documented by McCloy and Beck [12]. A review of the literature on cavitation in oil hydraulic systems is reported by Wiggert, et al. [13].

An experimental investigation was undertaken to study cavitation damage mechanisms in aircraft hydraulic systems. The purpose of the investigation was to identify mechanisms which lead to damage. A test facility was designed which allowed for the measurement of hydraulic parameters under both noncavitating and cavitating conditions. Diagnostic techniques were developed to detect the onset and the extent of cavitation. Cavitation inception, its definition, and the formulation of criteria are the subject of this paper.

\section{Test Facility}

Cavitation has been investigated in directional control valves in order to identify damage mechanisms characteristic of components of aircraft hydraulic systems. Tests have been 


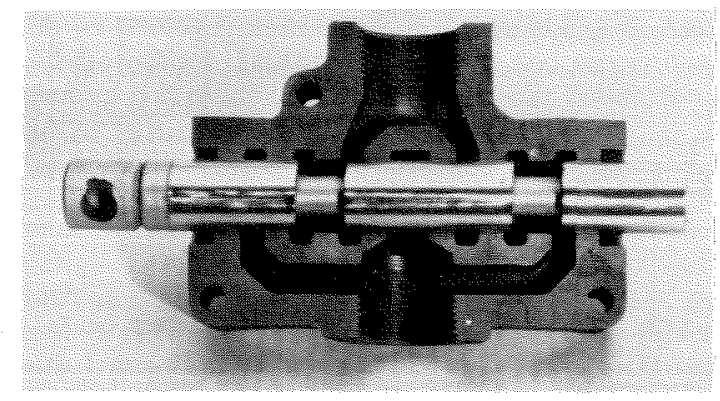

Fig. 1 Cutaway and spool of prototype valve

conducted in both a metal prototype valve and a plexiglass model three times its size. A common test facility was designed that allowed for control of flow, upstream pressure, downstream pressure, dissolved gas content, and spool position. In order to facilitate the continuous positioning of the spool, as well as the mounting of instrumentation on the spool itself, a simple industrial four-way lever-operated spool valve was chosen as the prototype. A cutaway of this valve, which has a spool diameter of $19.05 \mathrm{~mm}(0.75 \mathrm{in}$.$) , is shown in$ Fig. 1. In order to maximize the pressure differential across the valve, only one-half of the valve was used, resulting in effect a two-way valve.

The model valve was made of plexiglass with a spool diameter of $57.2 \mathrm{~mm}$ (2.25 in.). The size of the valve chamber, lands, and ports of the model valve were scaled up from the metal prototype valve. Figure 2 is a longitudinal view of the body of the model valve. The opening of the annular space could be controlled by the mechanism attached to the end of the spool. A similar mechanism was installed on the end of the prototype valve. The supply and return ports in the vicinity of the model spool were made to scale with the prototype valve.

The common hydraulic test loop consisted of an axial piston pump with a pressure regulator, a high pressure supply line, a flow meter, a bypass system, the particular test valve and its instrumentation, downstream valves for back pressure control, and a low pressure return line to the pump. A high pressure filter with an absolute rating of $3 \mu \mathrm{m}$ was in continuous use for control of contaminants. An auxiliary system consisting of a vacuum tank, tubing and valves, and a rubber bag storage reservoir was used to degas the oil. Oil was pumped from the closed bag through a nozzle on top of the vacuum tank and allowed to flow down the sides of the tank as a film. The total air content was monitored by use of an Aerometer, a commercial device similar to a Van Slyke ap-

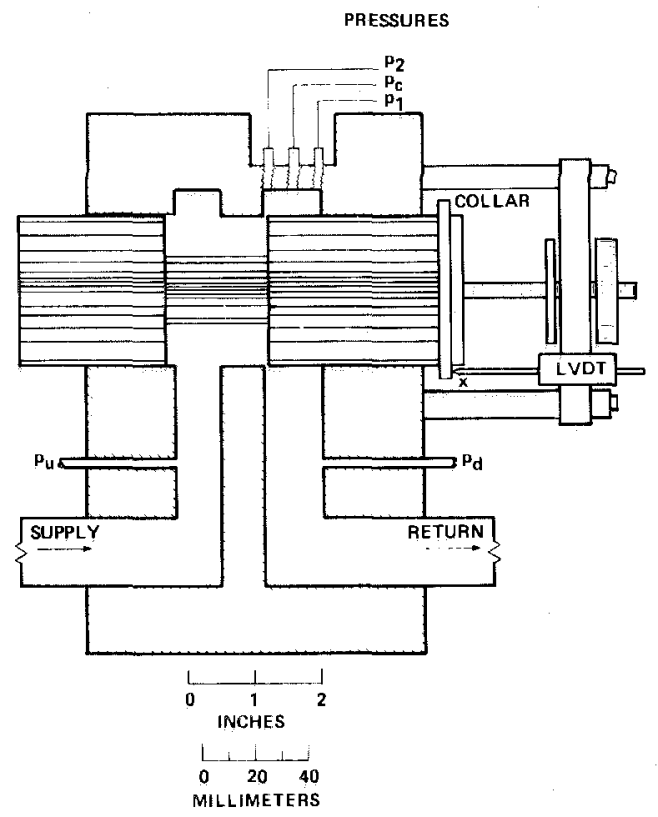

Fig. 2 Longitudinal section of model valve

paratus, which uses a filming technique to remove the gas. At room temperature and atmospheric pressure, the dissolved gas content of the oil employed in this investigation varied between 9.5 and 10 percent by volume. The minimum value achieved after several hours of degassing was 4 percent.

For the entire investigation, a petroleum-based hydraulic oil (MIL-H-5606C) was employed as the test liquid. A brief listing of some of the pertinent characteristics of this particular oil are:

Temperature $\left({ }^{\circ} \mathrm{C}\right) \quad$ Specific gravity Viscosity $(\mathrm{cS})$ Vapor pressure

$\begin{array}{llll}20 & 0.872 & 29.5 & 11.6 \\ 40 & 0.857 & 16.8 & 25.0 \\ 60 & 0.843 & 10.4 & 53.0\end{array}$

\section{Instrumentation and Data Acquisition}

For the steady-flow results reported here, mean flow rates and pressures were measured to characterize the test conditions, while dynamic pressures and acceleration were monitored to assess the onset and extent of cavitation.

\section{Nomenclature}

$$
\begin{aligned}
A & =\text { area of annular orifice } \\
A_{c} & =\text { area of vena contracta } \\
a & =\text { acoustic velocity } \\
B_{1}, B_{2}, B_{3} & =\text { coefficients } \\
C_{c} & =\text { coefficient of contraction } \\
C_{d} & =\text { coefficient of discharge } \\
c & =\text { radial distance between spool and wall } \\
D & =\text { spool diameter } \\
d & =\text { radial distance between spool neck and } \\
& \text { valve body } \\
\boldsymbol{E} & =\text { radial eccentricity between spool and valve } \\
f & =\text { body } \\
K & =\text { coefuency } \\
l & =\text { axial distance along jet } \\
p & =\text { pressure } \\
p^{\prime} & =\text { fluctuating pressure } \\
p_{1} & =\text { pressure at transducer } P_{1} \\
p_{2} & =\text { pressure at transducer } P_{2}
\end{aligned}
$$

$$
\begin{aligned}
p_{c} & =\text { mean pressure in chamber } \\
p_{L} & =\text { load (upstream) pressure } \\
p_{R} & =\text { return (downstream) pressure } \\
p_{v} & =\text { vapor pressure } \\
t & =\text { Reynolds number } \\
u_{0}^{\prime} & =\text { fluctuating velocity on jet centerline } \\
V_{c} & =\text { velocity in vena contracta } \\
V_{j} & =\text { jet velocity } \\
V_{0} & =\text { approach velocity } \\
x & =\text { axial position of valve spool } \\
\ddot{x} & =\text { acceleration of spool } \\
\Delta f & =\text { width of frequency channel } \\
\Delta p=p_{L}-p_{R} & =\text { pressure differential across valve } \\
\mu & =\text { dynamic viscosity of liquid } \\
\nu & =\text { kinematic viscosity of liquid } \\
\rho & =\text { mass density of liquid } \\
\sigma=p_{c}-p_{v} / \Delta p & =\text { cavitation index }
\end{aligned}
$$


Mean Quantities. For both test valves, a drag-type flow meter was used to measure the flow rate. This device consists of a circular target mounted on the end of a strain-gage instrumented cantilever beam. A resistance detector was used to monitor the oil temperature immediately upstream of the test valve in question. An accurate determination of the position of the specific valve was essential because of the relative small openings in practice. For each valve, a linear variable displacement transducer (LVDT) was used to measure the relative position $x$ of the spool.

Pressure transducers were used to measure mean upstream (load $\left.-p_{L}\right)$, differential $(\Delta p)$, chamber $\left(p_{c}\right)$, and downstream (return $-p_{R}$ ) pressures. Figure 2 shows the location of the pressure taps for the model valve. In this case, the differential pressure is defined by $p_{L}-p_{R}$. For the model, the load pressure $p_{L}$ was not measured directly. The load, return, and differential pressure were measured by either strain-gage or variable reluctance diaphragm pressure transducers. The mean chamber pressure $p_{c}$ for each valve was sensed by semiconductor pressure transducers. As shown in Fig. 2, transducer $\boldsymbol{P}_{c}$ is located exactly in the middle of the model chamber. For the prototype valve, the chamber transducer is located somewhat downstream of the center of the port.

Dynamic Quantities. Fluctuating pressures in the chamber of each valve were measured for the detection of cavitation by high-frequency response piezoelectric pressure transducers. These transducers were flush mounted in the chamber of each valve, as shown in Fig. 2 for the model valve. The diameter of the sensing surface is $2.51 \mathrm{~mm}(0.099 \mathrm{in}$.). The transducers had a resonant frequency in air of $250 \mathrm{kHz}$. The larger size model allowed for the installation of two piezoelectric transducers, referred to as $P_{1}$ and $P_{2}$ on Fig. 2 . The single piezoelectric transducer in the prototype valve chamber is called $P_{1}$. This transducer is located somewhat downstream of the middle of the chamber.

For the expected detection of mechanical effects in the spool related to cavitation, a high-frequency response quartz accelerometer was mounted on the end of the spool of the prototype valve. The miniature accelerometer had an undamped natural frequency in air of $120 \mathrm{kHz}$.

Data Acquisition System. The data acquisition system used to sample the mean and dynamic signals will be described with reference to Fig. 3. The test valve shown in the illustration could either be the model or the prototype valve. For the model valve, there was no measurement of load pressure $p_{L}$, nor any accelerometer signal $\ddot{x}$. On the other hand, for the prototype valve, usually only one piezoelectric pressure transducer $\left(P_{1}\right)$ was used.

The central device regarding the collection of mean quantities to characterize the flow conditions is a desktop computer. This computer controlled a multiplexer and a $61 / 2$ digit voltmeter. The analog signal from any of the six input channels shown in Fig. 3 could be transferred through the multiplexer by the controller to the voltmeter, which digitized the signal and transferred the data to the computer. For a given channel, 20 samples were averaged to obtain a mean value. At the completion of the entire test series, the data were then stored on a cassette tape of the desktop computer for future retrieval and analysis.

As illustrated on Fig. 3, the dynamic quantities monitored in this investigation are the fluctuating pressures $p_{1}$ and $p_{2}$ and the fluctuating spool acceleration $\ddot{x}$. Any two of these signals could be simultaneously input into the signal analyzer, which is an all-digital instrument capable of providing both time and frequency domain analysis of complex analog signals in the range of d-c to $25.6 \mathrm{kHz}$. Although it is recognized that cavitation noise usually exists at frequencies greater than $25.6 \mathrm{kHz}$, no data were collected beyond this limit because of equipment limitations.

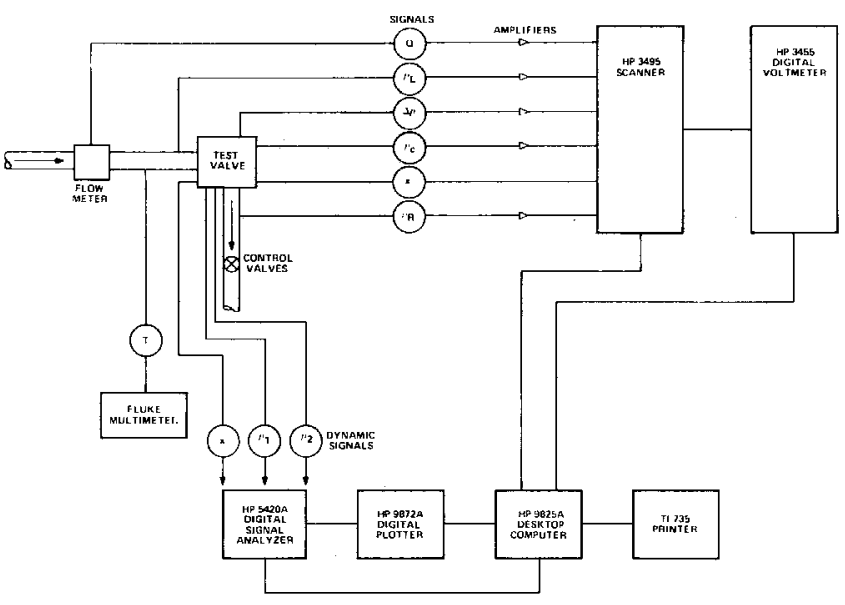

Fig. 3 Data acquisition system

Table 1 Estimates of experimental uncertainty

\begin{tabular}{|c|c|c|}
\hline Quantity and units & Mean value & Fluctuating value \\
\hline $\begin{array}{l}\text { Flow rate }\left(\mathrm{m}^{3} / \mathrm{s}\right) \\
\text { Valve position }(\mathrm{m}) \\
\text { Temperature }\left({ }^{\circ} \mathrm{C}\right) \\
\text { Pressure }(\mathrm{Pa}) \\
\text { Acceleration }(\mathrm{g})\end{array}$ & $\begin{array}{l}=1 \times 10^{-5} \\
\pm 5 \times 10^{-6} \\
=0.5 \\
\pm 2 \times 10^{4} \\
--\end{array}$ & $\begin{array}{c}-- \\
-- \\
-- \\
\pm 2 \\
\pm 5 \times 10^{-5}\end{array}$ \\
\hline
\end{tabular}

Test Procedure. Although occasionally the cross spectrum and coherence between $P_{1}$ and $P_{2}$ for the model or $P_{1}$ and $\ddot{x}$ for the prototype valve were measured, the most frequent and useful measurements were the auto power spectra for the two channels. The frequency resolution or $\Delta f$ for the analysis was $1 / 256$ of the bandwidth, or $100 \mathrm{~Hz}$. The signals were analyzed using a modified Hanning-type window. The power spectral density function for the auto spectra will be in units of $\sqrt{\overline{p^{\prime 2}} /}$ $\Delta f$ for the pressure transducers and $\left[\overline{\left(d^{2} x^{\prime} / d t^{2}\right)^{2}}\right]^{1 / 2} / \Delta f$ for the accelerometer. The total energy over any frequency range could be quickly extracted from the analyzer, facilitating the real-time analysis and the establishment of criteria for cavitation inception.

Experimental Uncertainty. The flow meter, LVDT, and the seven pressure transducers used to measure mean pressures in the two valves were all calibrated in situ using the same signal conditioning and data acquisition system as employed in the dynamic studies. For each of these instruments, from 8-20 calibration points were analyzed using a least-square regression technique. The manufacturer's calibration constants were used for the temperature detector, the accelerometer, and the three piezoelectric pressure transducers. For all instruments, the least-square analyses yielded a value of the coefficient of determination $r^{2} \geq 0.99$.

Considering all factors, the experimental uncertainties are as listed in Table 1.

\section{Valve Characteristics}

The mean-flow characteristics of each valve were determined by direct measurement before embarking upon the main cavitation study. It was essential to know accurately the value of the discharge coefficient of the valve in question because of the difficulty in measuring the valve opening with the desired precision. Once the value of the discharge coefficient was known for the particular test conditions, the valve opening could then be calculated from the known flow rate and pressure difference across the valve, both of which could be measured quite accurately.

As demonstrated by McCloy and Beck [3], for certain Reynolds numbers free streamline theory can be applied to the 
annular jet issuing from the orifice shown in Fig. 4. Assuming inviscid flow from the approach region and recognizing that the opening $b$ is much less than the annular dimension $d$, the jet velocity in the vena contracta is defined by

$$
V_{c}=\sqrt{\frac{2 \Delta p}{\rho}}
$$

in which $\rho$ is the fluid density, and $\Delta p=p_{L}-p_{R}$, the nominal pressure difference measured by the differential pressure transducer. The volumetric flow rate through the valve is given by

$$
Q=A_{c} V_{c}=C_{c} \pi D b V_{c}
$$

in which $A_{c}$ is the area of the contracted jet, which is usually defined in terms of the area of the annular orifice, $C_{c}$ is the contraction coefficient, and $D$ is the diameter of the spool. The resulting relationship for the flow rate is

$$
Q=C_{d} \pi D b \sqrt{\frac{2 \Delta p}{\rho}}
$$

in which $C_{d}$ is the discharge coefficient and, in the case for which $b<<d$, is equal to $C_{c}$. The preceding equation suggests a linear relationship between $Q$ and $b$.

Because of the relatively small values of $b$ employed in this study, tests were instead conducted by varying $b$ while holding $\Delta p$ constant. In this instance, a slightly modified coefficient of discharge could be computed from

$$
C_{d}=\frac{d Q / d x}{\pi D \sqrt{\frac{2 \Delta p}{\rho}}}
$$

in which $x$ is the axial position of the spool as measured by the LVDT. Tests conducted at a constant $\Delta p$ and varying $x$ yielded a linear relationship between $Q$ and $x$, allowing for the determination of $C_{d}$ from the slope of the curve.

In general, it would be expected that $C_{d}$ would be a function of the Reynolds number and a cavitation index. For simplicity, the Reynolds number is defined on the basis of the average velocity at the annular opening instead of in terms of $V_{c}$ at the vena contracta. The length dimension chosen in the definition is $b$, yielding

$$
R=\frac{\rho(Q / A) b}{\mu}=\frac{Q}{\pi D \nu}
$$

in which $\mu$ and $\nu$ are the dynamic and kinematic viscosities of the liquid, respectively. For a given temperature, equation (5) suggests that the Reynolds number is independent of the valve opening $b$ at a constant discharge.

The cavitation index is normally defined as the ratio of a reference pressure minus the vapor pressure of the liquid to a dynamic pressure, the latter of which is referenced to the approach velocity $V_{0}$. A more appropriate dynamic pressure for the spool valve would be that based on the jet velocity $V_{j}$ which is not measured directly, but instead directly related to $\Delta p$. The cavitation number is defined as

$$
\sigma=\frac{p_{c}-p_{\nu}}{\Delta p}
$$

In general, it would be expected that the discharge coefficient would depend upon both $R$ and $\sigma$, or

$$
C_{d}=C_{d}(R ; \sigma)
$$

Extensive testing over a range of $R$ from 150 to 1500 under noncavitating conditions showed that $C_{d}$ was virtually independent of viscous effects, yielding an average value of $C_{d}$ equal to 0.68 for the prototype valve and 0.66 for the model

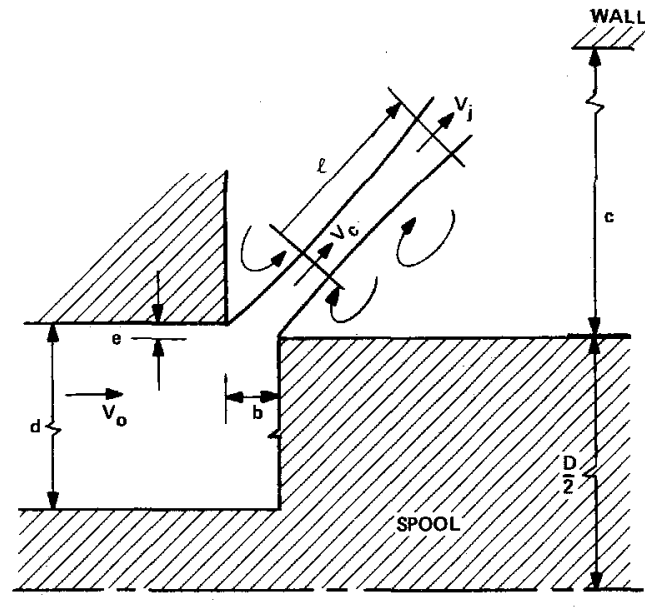

Fig. 4 Definition of valve and jet characteristics

valve. These values, which are reported in Martin, et al. [14], correspond closely to the theoretical value of 0.677 calculated by von Mises using two-dimensional inviscid theory. A summary of the theory is reported by Robertson [15]. Experimental results published by McCloy and Beck [3] show a similar trend. Apparently the Reynolds number defined by equation (5) must be less than approximately 100 before viscous effects commence to influence the mean flow characteristics of the valve.

As shown by a number of researchers in the study of cavitation in pipe orifices, cavitation has to become quite extensive before there is any effect on $C_{d}$. For the noncavitating and moderate cavitation reported in this paper, it will suffice to use the constant values of 0.68 (prototype valve) and 0.66 (model valve) in any calculations of $b$ from equation (3). Further details on the effect of $R$ and $\sigma$ on $C_{d}$ may be found in the report by Martin, et al. [14].

\section{Noncavitating Spectra}

Prior to the establishment of criteria for the inception of cavitation, the level of fluctuations of pressure and acceleration had to be understood for noncavitating flow in each valve. Extensive testing was conducted to correlate energy levels of pressure fluctuations with flow rate and valve opening. Care was taken to insure that the chamber pressure $p_{c}$ was large enough to inhibit any cavitation. This was accomplished by monitoring the total mean-square energy over the entire spectra for various values of $\sigma$. It was found that, for a constant discharge $Q$ and valve opening $b$, the total energy did not vary if $\sigma$ was above a certain threshold value, to be discussed later. The tests under noncavitating conditions were also of value in observing changes in the jet flow pattern for different conditions.

The pressure transducers $P_{1}$ and $P_{2}$ in the model and $P_{1}$ in the prototype chamber experience the acoustical noise from the confined jet issuing from the annular orifice. The fact that the discharge coefficient is constant over the range of Reynolds numbers tested would suggest a turbulent jet. As shown by McCloy and Beck [12] in their study of jet hysteresis, the jet can either reattach on the surface of the spool or on the wall of the port, or issue freely. A shifting jet pattern was clearly indicated in the model chamber by observing the energy levels on the two transducers, which were located at the extremities of the cavity.

The dynamics of an unconfined plane jet issuing from a nozzle are fairly well documented, albeit at higher Reynolds numbers than experienced with the two valves. As experimentally shown by Albertson, et al. [16], and later by Gutmark and Wygnanski [17], the mean centerline velocity of a plane jet decreases as the square root of the ratio of the 
initial jet diameter to the axial distance. In terms of the definition sketch on Fig. 4

$$
\frac{V_{j}}{V_{c}}=K \sqrt{\frac{b}{l}}
$$

in which $K$ is a constant for plane jets at large Reynolds number and $l$ is the axial distance from the nozzle, or from the vena contracta in the case of an orifice. For a plane turbulent jet, Gutmark and Wygnanski [17] found that the rms value of the fluctuating velocity $u_{0}^{\prime}$ on the centerline of the jet was a constant when normalized with $V_{j}$, or

$$
\sqrt{\frac{u_{0}^{\prime 2}}{V_{j}}}=\text { constant }
$$

Reethof [18] states that a fluctuating mass flow rate with inphase pressure fluctuations would correspond to a monopole source, for which the rms energy of fluctuating pressure in this case are assumed to be correlated by

$$
\sqrt{\overline{\bar{p}^{\prime 2}}}=B_{1} \rho V_{j}^{2}
$$

in which $B_{1}$ is a dimensionless coefficient which would depend upon transducer location, Reynolds number, etc. If, instead the fluctuations emanate from dipole sources - which may be related directly to fluctuating pressures

$$
\sqrt{\overline{\mathrm{p}^{\prime 2}}}=B_{2} \rho V_{j}^{2}\left(\frac{V_{j}}{a}\right)
$$

in which $a$ is the acoustic velocity.

For a monopole source, equation (10) along with the jet relationships (2) and (8) yield the mean-square energy

$$
\overline{p^{\prime 2}}=B_{1}^{2}\left[\frac{K}{\pi C_{c}}\right]^{4} \frac{\rho^{2}}{D^{4} b^{2} l^{2}} Q^{4}
$$

In the case of dipole sources

$$
\overline{p^{\prime 2}}=B_{2}^{2}\left[\frac{K}{\pi C_{c}}\right]^{1} \frac{\rho^{2} Q^{6}}{\overline{D^{6} b^{3} l^{3} a^{2}}}
$$

In the following discussion of the slope of the energy spectra and the correlation of mean-square energy with the flow rate, a direct comparison of the results for the two valves is somewhat subjective because of the size of the pressure transducer relative to the respective valve chamber dimensions, which was three times greater in the prototype valve than in the model valve. For certain jet flow, patterns there are, however, striking similarities between the noncavitating spectra for the two valves.

All noncavitating spectra measured by transducer $P_{1}$ in the chamber of the prototype valve indicate that the mean-square energy can be correlated in terms of a dipole, or fluctuating pressure, source. The mean-square energies measured with this transducer correlate well with $Q^{6}$, as shown by the two data sets in Fig. 5. The data shown in Fig. 5 were taken at virtually the same opening $b$, but quite different Reynolds numbers. If $l$ is assumed to be fixed and to correspond to the distance from the orifice opening to transducer $P_{1}$, then equation (13) can be reduced to

$$
\overline{p^{\prime 2}}=B_{3} \rho^{2} Q^{6}
$$

as $a, D$, and $b$ are virtually constant. Although there is a Reynolds number variation for each data set, the coefficient $B_{3}$ appears to be a constant for each. Energy spectra for two of the data points plotted on Fig. 5 are presented in Fig. 6. The slope of each curve can be approximated by -1 for the

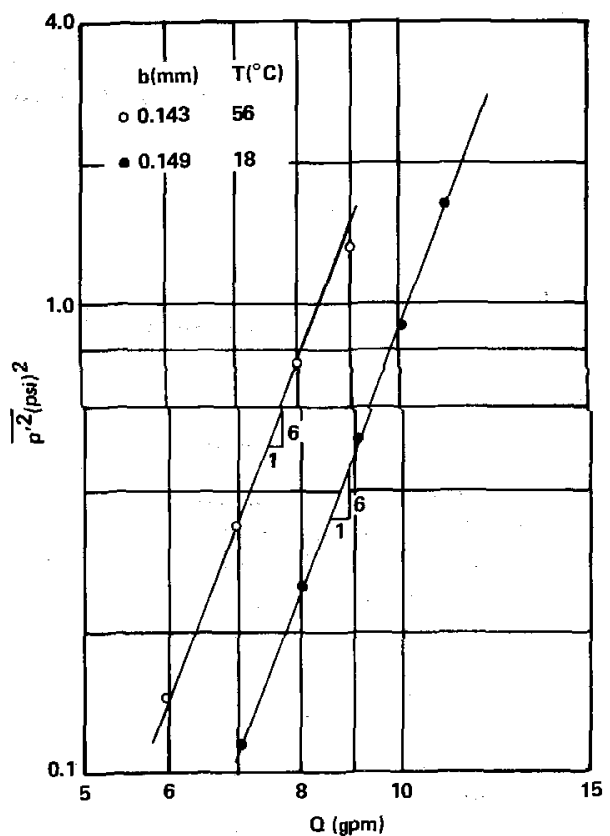

Fig. 5 Noncavitating mean-square energy versus discharge for prototype valve at a tixed opening $(1 \mathrm{psi}=8895 \mathrm{~Pa}, 1 \mathrm{gpm}=6.31 \times$ $10^{-5} \mathrm{~m}^{3}$ (s)
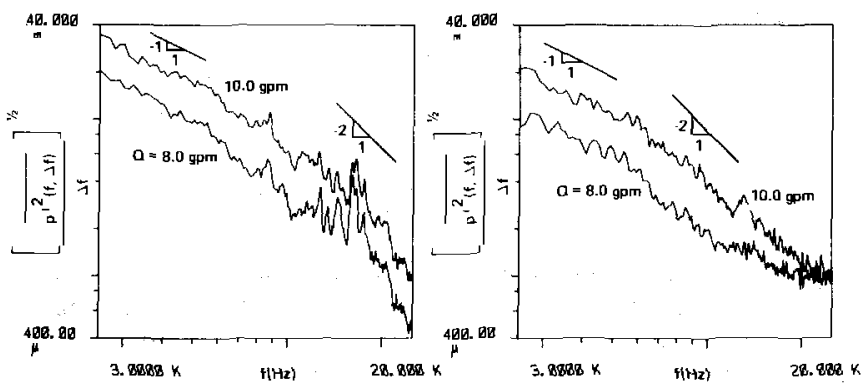

a.) $\mathrm{b}=0.143 \mathrm{~mm}$ and $\mathrm{T}=56^{\circ} \mathrm{C}$

b.) $b=0.149 \mathrm{~mm}$ and $T=18 \mathrm{C}$

Fig. 6 Noncavitating energy spectra for prototype valve at a fixed opening $\left(1 \mathrm{psi}=6895 \mathrm{~Pa} ; 1 \mathrm{gpm}=6.31 \times 10^{-5} \mathrm{~m} / \mathrm{s}\right)$

lower frequency range and -2 for the higher frequencies. As demonstrated by Lush [19], a dipole source will produce energy spectra with a -1 slope. As most of the energy is in the first half of the frequency range shown in Fig. 6, the dipole source dominates, resulting in a relationship represented by equation (13). An examination of many noncavitating spectra shows that, as the flow rate and hence jet velocity $V_{j}$ is increased for a fixed opening $b$, the extent of the spectrum corresponding to a -1 slope increases. Indeed, for high frequencies, the values of the noncavitating spectra approach the background noise level, which was $6.9 \mathrm{~Pa}(0.001 \mathrm{psi})$ for the pressure transducer in the prototype valve.

The two pressure transducers in the model were located such that any significant changes in the jet orientation could be noticed. The noncavitating results presented here suggest that the jet did flip back and forth, confirming the conclusions of McCloy and Beck [12] that the jet may reattach to either the spool surface or the port wall, or issue freely at an angle depending upon the opening $b$ and any clearance between the spool and the land of the valve body.

The variation of mean-square energy with flow rate is shown in Fig. 7 for various openings of the model valve. The pressure difference $\Delta p$ was maintained constant under noncavitating conditions. For a free jet for which $\Delta p$ is a constant, the velocity at the vena contracta $V_{c}$ as given by equation (1) should have been a constant for all of the data shown in Fig. 7. According to equation (8), the jet velocity at 


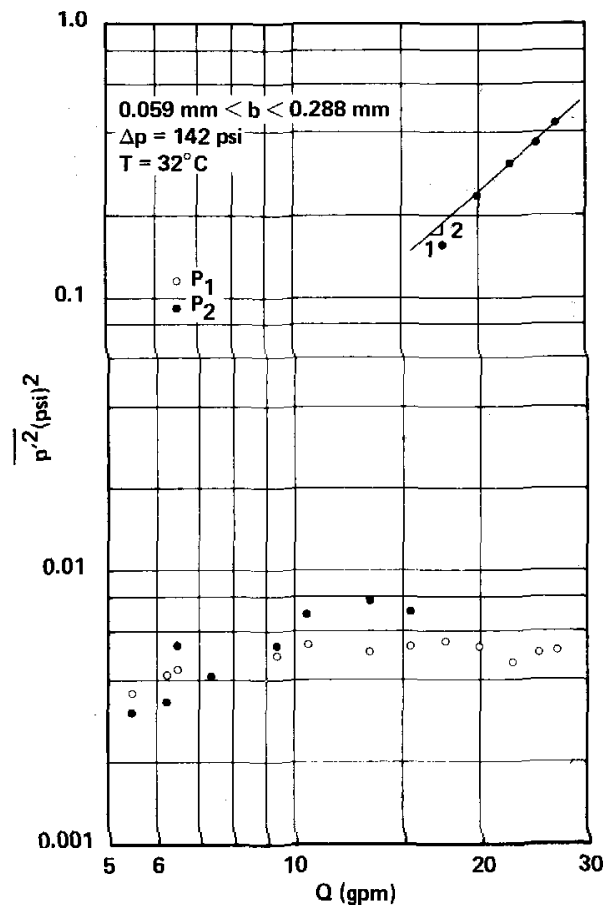

Fig. 7 Noncavitating mean-square energy versus discharge for model valve at a variable opening and cpnstant pressure difference (1 psi = $6895 \mathrm{~Pa} ; 1 \mathrm{gpm}=6.31 \times 10^{-5} \mathrm{~m}^{3} / \mathrm{s}$ )
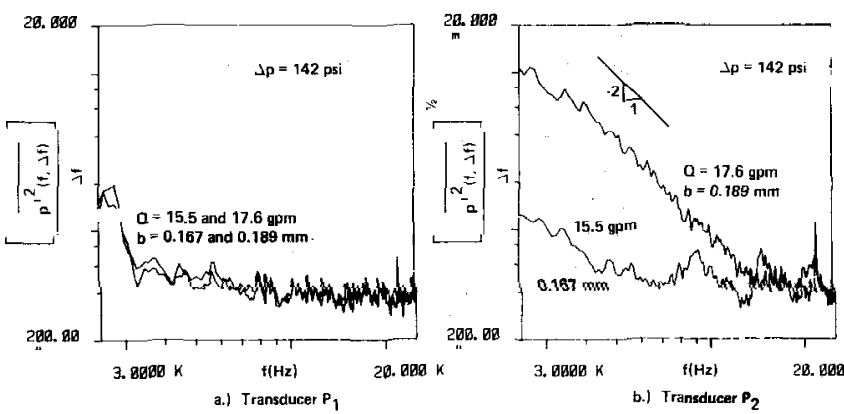

Fig. 8 Noncavitating energy spectra for model valve at two values of opening $b$ and constant pressure difference $(1 \mathrm{psi}=6895 \mathrm{~Pa} ; 1 \mathrm{gpm}=$ $\left.6.31 \times 10^{-5} \mathrm{~m}^{3} / \mathrm{s}\right)$

some position $l$ from the vena contracta would increase directly with the square root of the opening $b$. The nearly constant output from transducer $P_{1}$ over the range of openings would indicate that the jet was not directed toward $P_{1}$. For flows less than $9.78 \times 10^{-4} \mathrm{~m}^{3} / \mathrm{s}(15.5 \mathrm{gpm})$, the jet is probably between $P_{1}$ and $P_{2}$ because of the relatively low energy levels, which are only slightly greater than the background noise level. As shown by Fig. 7 and the corresponding spectra in Fig. 8 for transducer $P_{2}$, there is an apparent shift in the flow pattern as the flow increased slightly from $9.78 \times 10^{-4}$ to $11.2 \times 10^{-4} \mathrm{~m}^{3} / \mathrm{s}(15.5$ to $17.6 \mathrm{gpm})$. As the valve was opened further, the energy level sensed by $\boldsymbol{P}_{2}$ increased as the square of the flow, suggesting a monopole source in accordance with equations (3) and (12) for a variable opening $b$. It is suspected that the increase in energy is either due to direct impingement of a reattached jet or the nearly direct effect of a free jet. The slope of the energy spectra at location $P_{2}$ can be approximated by -2 over a range of frequencies.

Noncavitating data were collected with the model valve fixed at openings of $b=0.108$ and $0.237 \mathrm{~mm}$. For the smaller opening, the plot of mean-square energy versus flow rate shown in Fig. 9 and energy spectra for two of the higher flow rates plotted in Fig. 10 indicate that the jet may have been free, and furthermore directed toward transducer $P_{1}$. At the

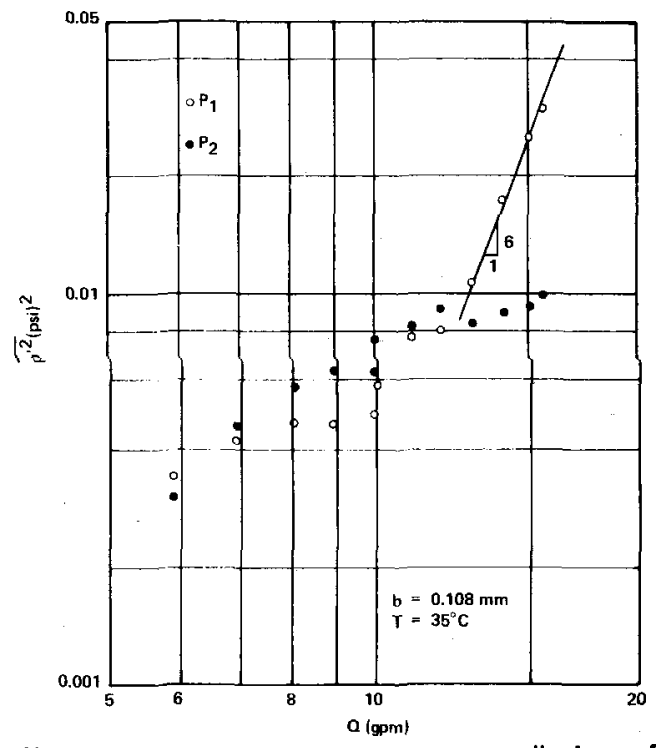

Fig. 9 Noncavitating mean-square energy versus discharge for mode valve at a fixed opening $\left(1 \mathrm{psi}=6895 \mathrm{~Pa} ; 1 \mathrm{gpm}=6.31 \times 10^{-5} \mathrm{~m}^{3} / \mathrm{s}\right)$
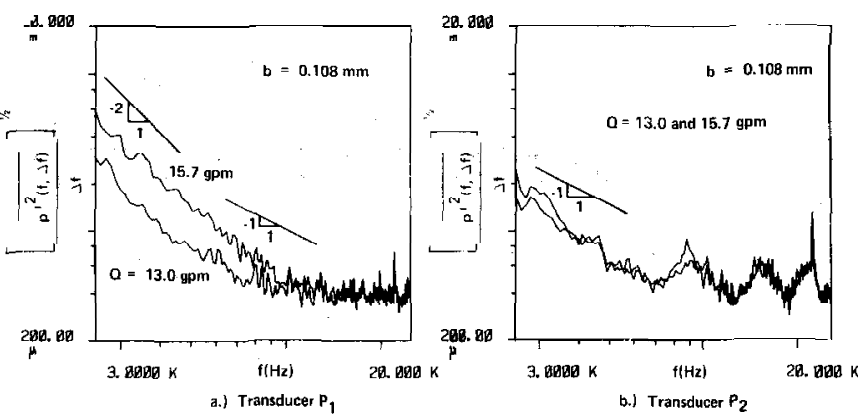

Fig. 10 Noncavitating energy spectra for model valve at a fixed opening ( 1 psi $\left.=6895 \mathrm{~Pa} ; 1 \mathrm{gpm}=6.31 \times 10^{-5} \mathrm{~m}^{3} / \mathrm{s}\right)$

greater opening, however, the transducer $P_{1}$ hardly experienced any change in energy fluctuations as the flow increased, as shown in Figs. 11 and 12(a). There is some indication that the slope of the mean-square energy versus flow rate correlation changes from a dipole source $(6$ to 1$)$ to a monopole source (4 to 1) at the higher flow rates. Portions of the jet-induced spectra plotted in Fig. $12(b)$ can be approximated by slopes of -1 , and others by -2 .

The direct effect of pressure fluctuations from a jet are clearly shown in Figs. 8(b), 10(a), and 12(b) for the model, in contrast to Figs. $8(a)$ and $12(a)$, for which there is little to no jet effect, but instead only background noise. As recently reviewed by Arndt [20], the eff fect of a turbulent shear flow on noncavitating pressure spectra has recently received attention by George [21], Fuchs and Michalke [22], and George and Beuther [23]. References on noise in an underwater environment are cited by Ross [24]. The effect of any shifting of the jet is more evident in the larger model than in the prototype valve because of the size of the piezoelectric transducers relative to the length of the valve chamber.

\section{Cavitating Spectra}

In order to define and determine the condition of cavitation inception, tests were conducted over a range of values of the cavitation number $\sigma$. Because of the sensitivity of pressure fluctuations to changes in jet velocity, care was taken to maintain the volumetric flow $Q$ constant and to hold the respective spool at a fixed position. As shown by the noncavitating energy spectra, slight differences in valve opening $b$ can lead to significant changes in energy levels if there is a 


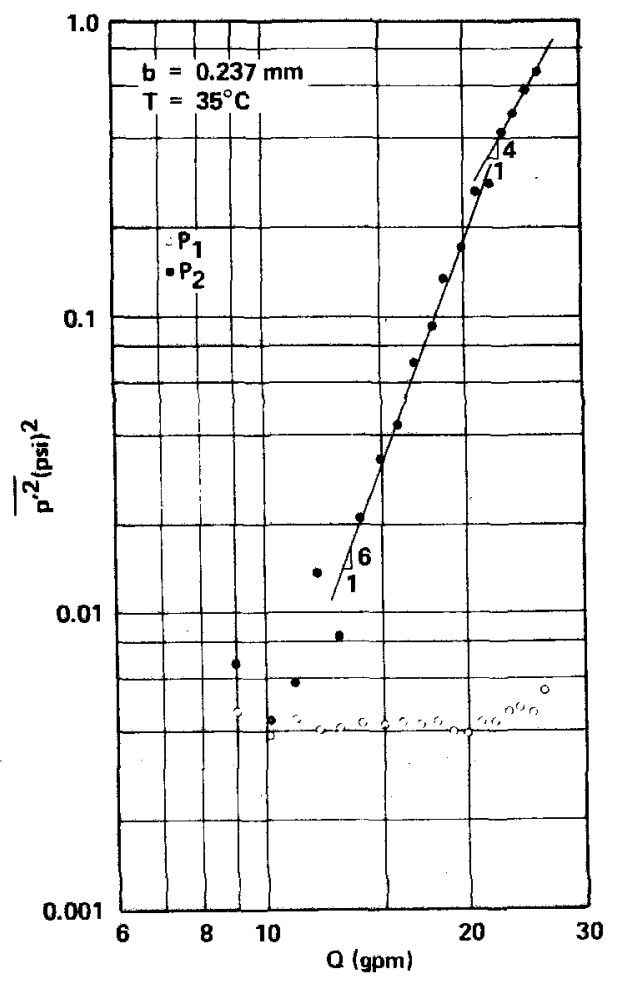

Fig. 11 Noncavitating mean-square energy versus discharge for model valve at a fixed opening ( $1 \mathrm{psi}=6895 \mathrm{~Pa} ; 1 \mathrm{gpm}=6.31 \times 10^{-5}$ $\left.m^{3} / s\right)$
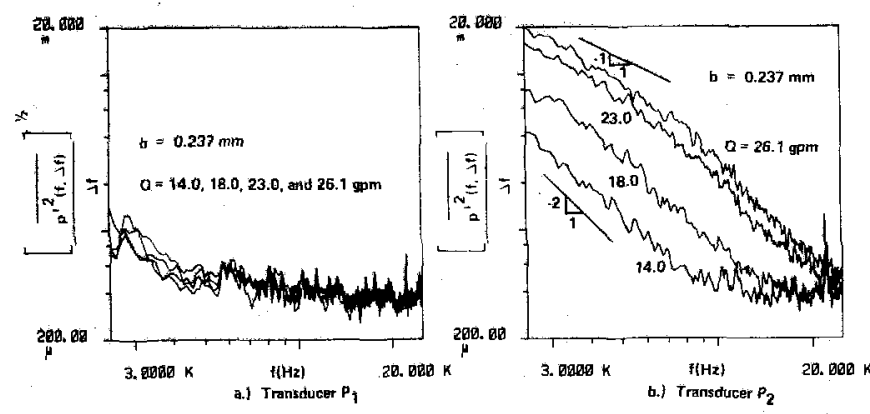

Fig. 12 Noncavitating energy spectra for model valve at a fixed opening $\left(1 \mathrm{psi}=6895 \mathrm{~Pa} ; 1 \mathrm{gpm}=6.31 \times 10^{-5} \mathrm{~m}^{3} / \mathrm{s}\right)$

direct effect of the jet. On the other hand, however, cavitation noise in the model valve can be several orders of magnitude greater than that due to a noncavitating jet.

Acoustical noise, pressure fluctuations, and accelerometer readings have been employed by numerous investigators over a span of years to detect cavitation inception and to correlate developed cavitation with the cavitation number. The effect of cavitation on the energy spectra measured by various instruments are reported by Jorgensen [25] for a cavitating submerged water jet, by Deeprose, et al. [26], for a cavitating pump, by Óba and Ito [27] for a cavitating venturi, and by Blake, et al. [28], for cavitation on a hydrofoil. For cavitation in orifices and water valves, Tullis and Govindarajan [29], and Ball and Tullis [30] used the rms accelerometer output as a criterion of defining cavitation inception, choking, etc. Tullis [31] later relates acceleration spectra with cavitation. In this investigation, it is shown that the use of rms energies must be applied with care in the definition of cavitation inception. It will be demonstrated that a more foolproof technique will be the careful study of energy spectra under similar geometric and flow conditions.

Prototype Valve. The results of varying $\sigma$ as $b$ and $Q$ are maintained nearly constant are presented in Fig. 13 for several
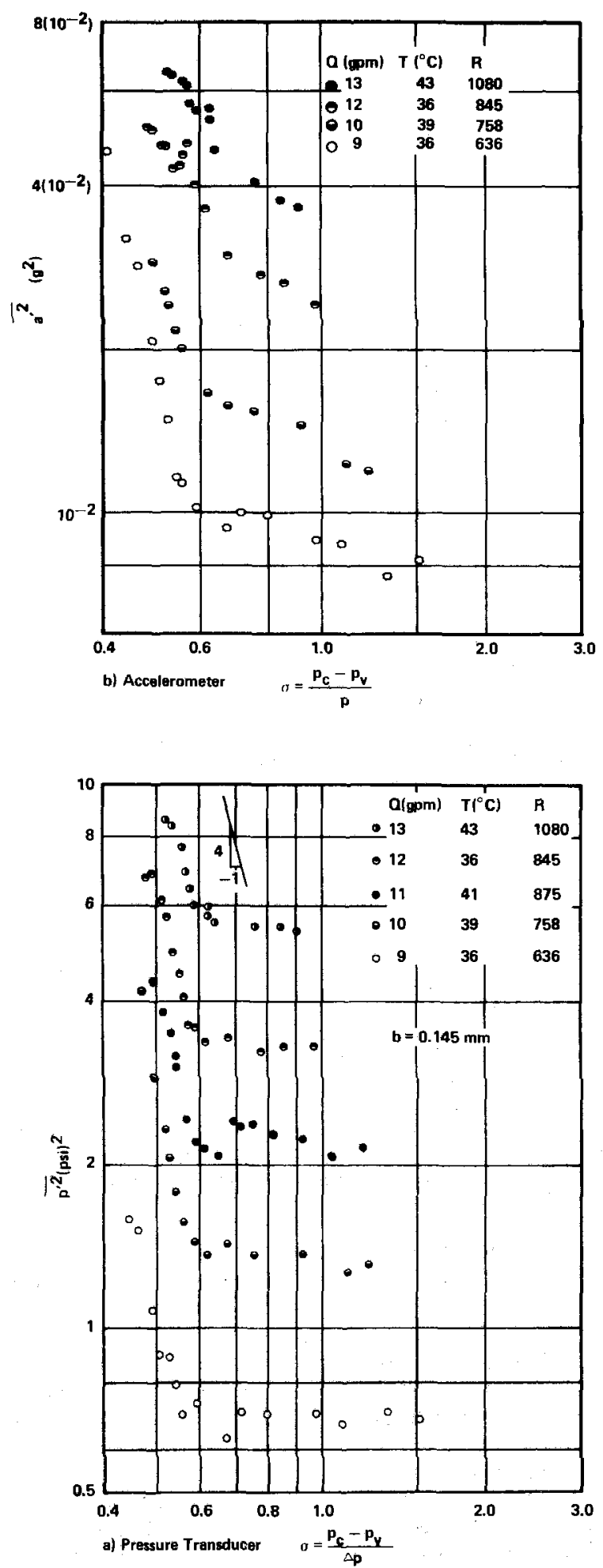

Fig. 13 Mean-square energy versus cavitation index for prototype valve at a fixed opening end constant discharges (1 psi $=6895 \mathrm{~Pa} ; 1$ $\left.g \mathrm{pm}=6.31 \times 10^{-5} \mathrm{~m}^{3} / \mathrm{s}\right)$

flow rates. Although for each flow rate there is eventually a continual increase in the fluctuating energy measured by the pressure transducer $P_{1}$ and the accelerometer $A_{1}$ as the chamber pressure $p_{c}$ is lowered, it is not clear where cavitation inception occurs. The definition of incipient cavitation is especially difficult with the prototype valve because of the relatively large jet energy under noncavitating conditions. In order to illustrate this difficulty, the data points for $Q=6.94$ $\times 10^{-4} \mathrm{~m}^{3} / \mathrm{s}(11 \mathrm{gpm})$ are plotted on Figs. $14(a)$ and $(b)$ at a 

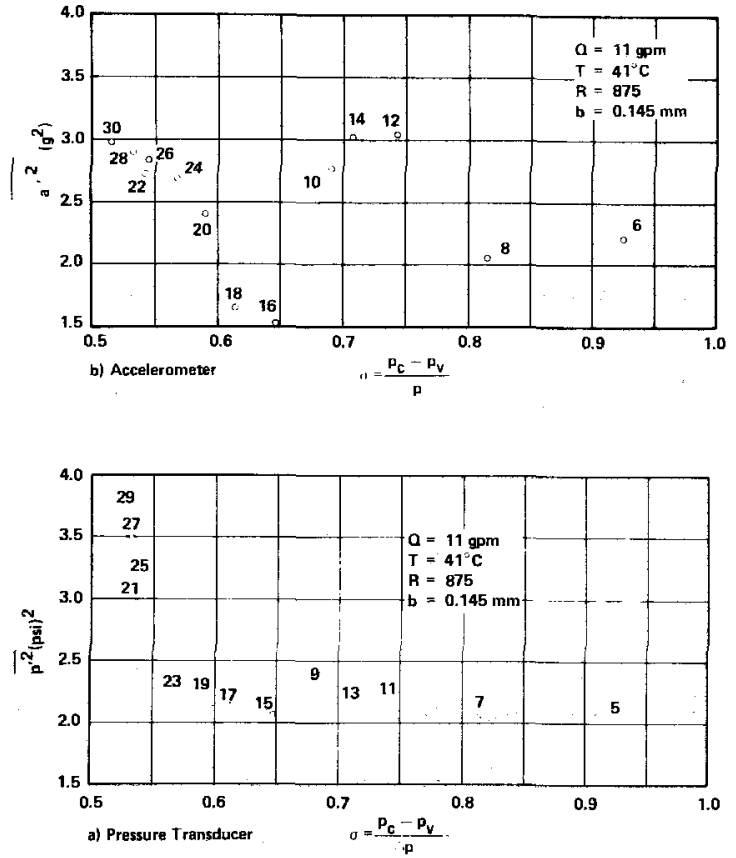

Fig. 14 Mean-square energy versus cavitation index for prototype valve at a fixed opening and $O=11 \mathrm{gpm}(1 \mathrm{psi}=6895 \mathrm{~Pa} ; 1 \mathrm{gpm}=$ $\left.6.31 \times 10^{-5} \mathrm{~m}^{3} / \mathrm{s}\right)$

different scale. For reference, each data point is given a number which actually corresponds to a data file on a storage tape. The definition of cavitation inception would be very difficult if not impossible on the basis of mean-square energy, because of the sudden decrease in energy level from point 9 to point 15 for $P_{1}$ and corresponding points 10 to 16 for the accelerometer. It was discovered that the respective energy spectra for each test proved more revealing that the total energy in the entire spectrum. In Figs. 15(a) $\left(P_{1}\right)$ and $15 b$ $\left(A_{1}\right)$, individual spectra for the seven points for which $\sigma>$ 0.6 are superimposed on the left while the spectra for two or three values of $\sigma$ are plotted on the right. The coincidence of the data on the left plot of pressure spectra suggests only noncavitating jet noise. Apparently few if no cavitation events occurred for $\sigma=0.614$ (17 and 18) but many were present once the cavitation number was slightly reduced to 0.591 (19 and 20). Due to the relatively high energy levels at the low frequency end of the spectrum resulting from jet noise, small increases in the spectral density at the higher frequencies as a result of limited cavitation do not contribute much to the total energy over the entire pressure spectrum. The parameter total energy becomes more indicative of cavitation levels once cavitation becomes more extensive. Although the acceleration spectra under noncavitating conditions are not as similar as the pressure spectra, it is fairly evident at high frequencies that cavitation has occurred between $\sigma=0.614$ and 0.591 .

The spectra in Fig. 15 indicate that cavitation noise is restricted to the higher frequencies, but extends over a wider band as the cavitation number decreases. Another measure of cavitation noise is the ratio of the power spectral density of each frequency channel $\Delta f$ under cavitating conditions to that under noncavitating conditions. Choosing points 15 and 16 $\left(\sigma_{0}=0.647\right)$ as the noncavitating reference values, the ratio of power spectral densities are plotted in Fig. 16 for points 17 and $18(\sigma=0.614)$ and points 19 and $20(\sigma=0.591)$. Additional ratios of the power spectral density are presented in Fig. 16 for lower values of the cavitation index, showing a progressive expansion of the cavitation noise to lower frequencies as $\sigma$ is decreased. A sensitive measure of the extent of cavitation noise is the area under the curve of the ratio of power spectral density versus frequency, as shown on Fig. 17
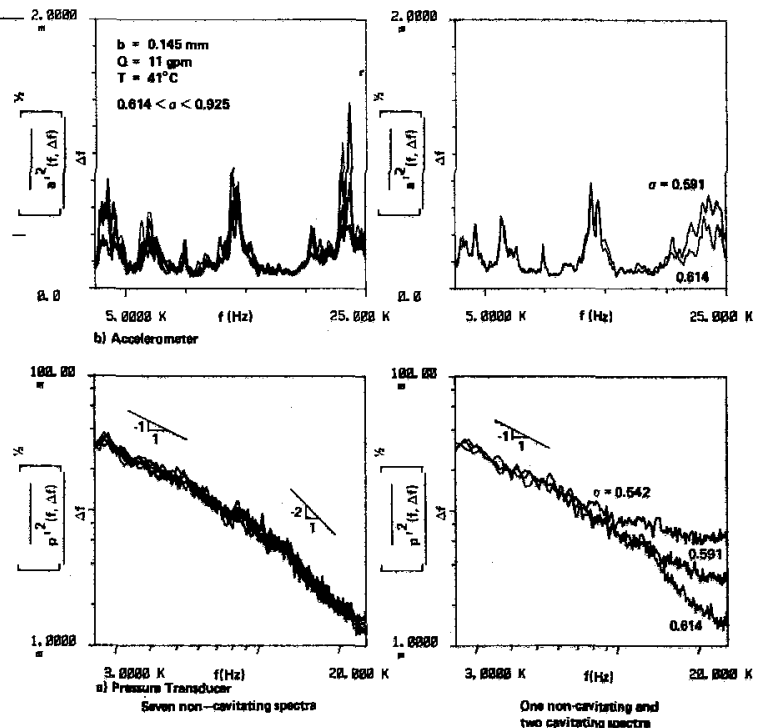

Fig. 15 Noncavitating and cavitating energy spectra for prototype valve at a fixed opening and $Q=11 \mathrm{gpm}(1 \mathrm{psi}=6895 \mathrm{~Pa} ; 1 \mathrm{gpm}=$ $\left.6.31 \times 10^{-5} \mathrm{~m}^{3} / \mathrm{s}\right)$
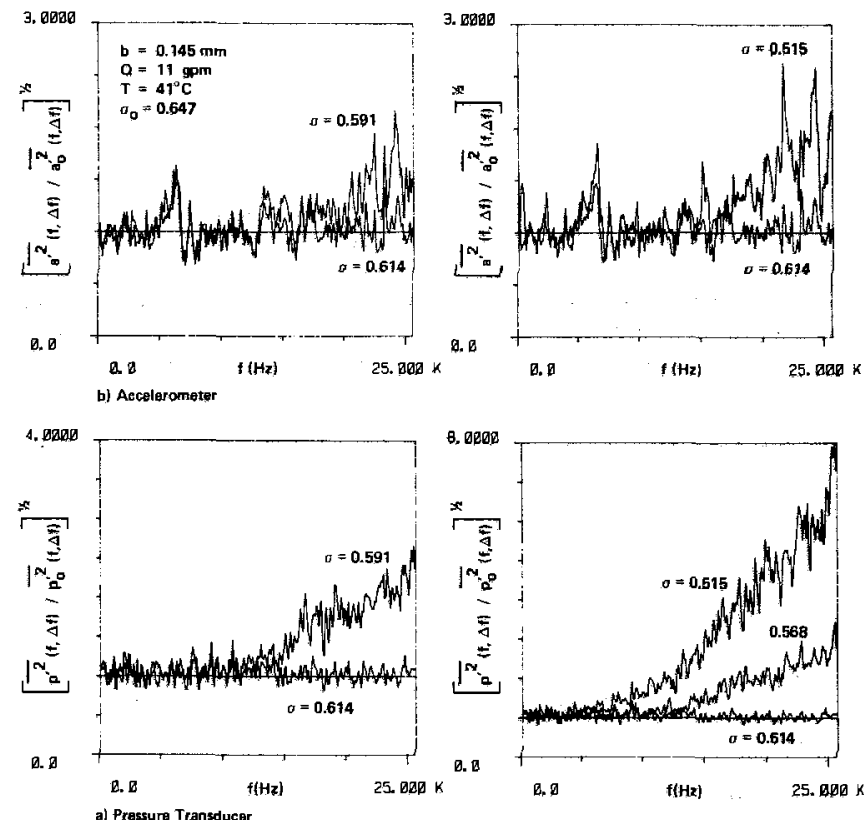

Fig. 16 Ratio of cavitating to noncavitating $\left(\sigma_{0}=0.647\right)$ spectra density for prototype valve at a fixed opening (1 gpm $=6.31 \times 10^{-5}$ $\left.\mathrm{m}^{3} / \mathrm{s}\right)$

for transducer $P_{1}$ for a flow of $Q=7.57 \times 10^{-4} \mathrm{~m}^{3} / \mathrm{s}(12$ gpm). Similar correlations occurred for other flow rates. In comparing the sensitivity of the piezoelectric pressure trans. ducer with the accelerometer, it is clear that both sense the onset of cavitation, but the pressure transducer is probably a better detector for inception because (1) it is located close to the source and (2) the accelerometer is more sensitive to valve noise from the downstream throttling valves, especially for large values of $p_{R}$ and hence $\sigma$.

Model Valve. Noncavitating and cavitating spectra for the model valve are shown in Fig. 18 for pressure transducers $\boldsymbol{P}_{1}$ and $P_{2}$. The six spectra shown on the left plots suggest no cavitation for $\sigma \geq 0.385$. As shown by the plots on the right figure, a slight lowering of $\sigma$ to 0.373 resulted in an increase in the power spectrum at the higher frequencies. The ratio of the power spectral density for the same data shown in Fig. 19 is 


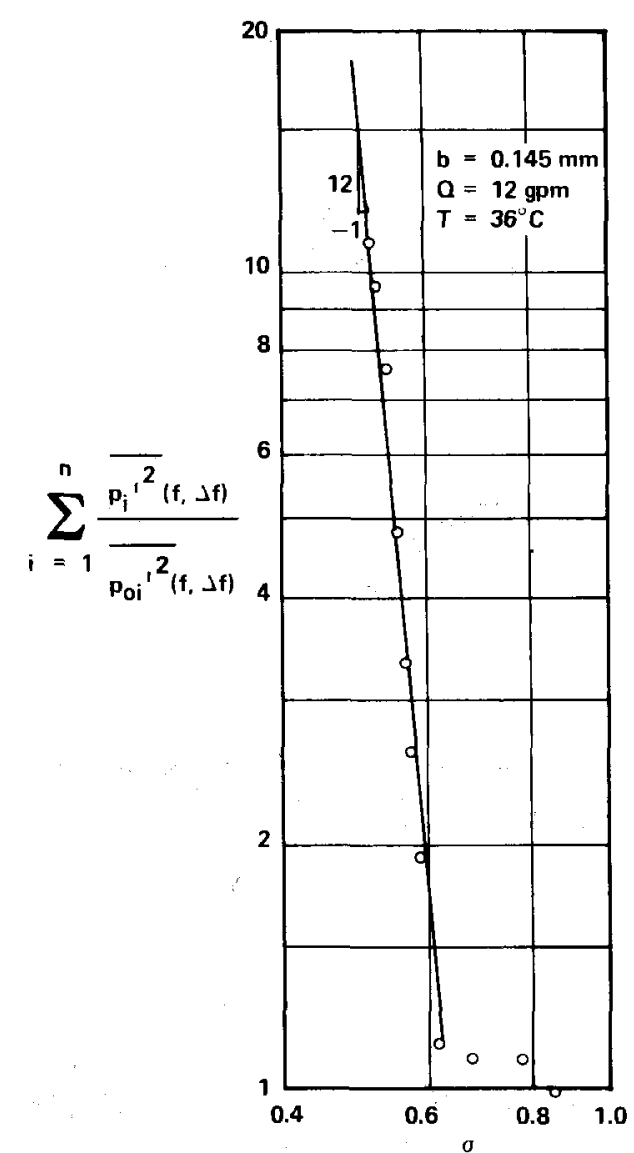

Fig. 17 Area under ratio of cavitating to noncavitating mean-square spectral density curve for fluctuating pressure versus cavitation index for prototype valve at a fixed opening and $Q=12 \mathrm{gpm}(1 \mathrm{gpm}=6.31 \times$ $\left.10^{-5} \mathrm{~m}^{3} / \mathrm{s}\right)$
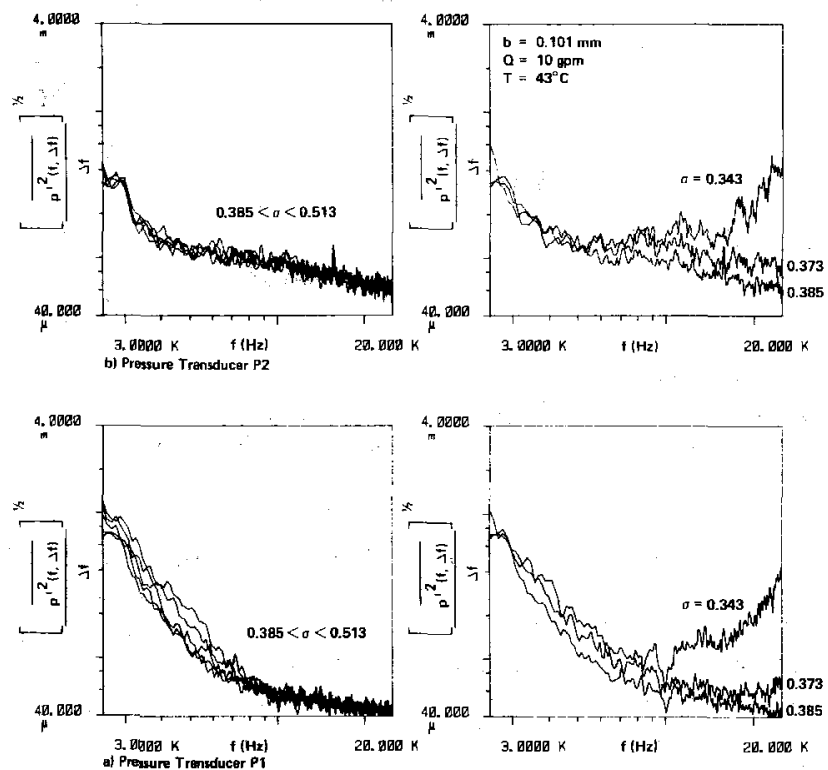

Fig. 18 . Noncavitating and cavitating energy spectra for model valve at a fixed opening (1 psi $\left.=6895 \mathrm{~Pa} ; 1 \mathrm{gpm}=6.31 \times 10^{-5} \mathrm{~m}^{3} / \mathrm{s}\right)$

also quite revealing regarding both the coincidence of noncavitating results, as well as the onset of cavitation. In fact, as illustrated by Fig. 20 for these data set and by Fig. 21 for another data set taken at a higher flow, the area under the curve of the ratio of power spectral density at any condition to a reference noncavitating condition is quite sensitive to cavitation events. Over a range of $\sigma$ of 0.15 in Fig. 20, the area function varied by nearly four orders of magnitude. For the
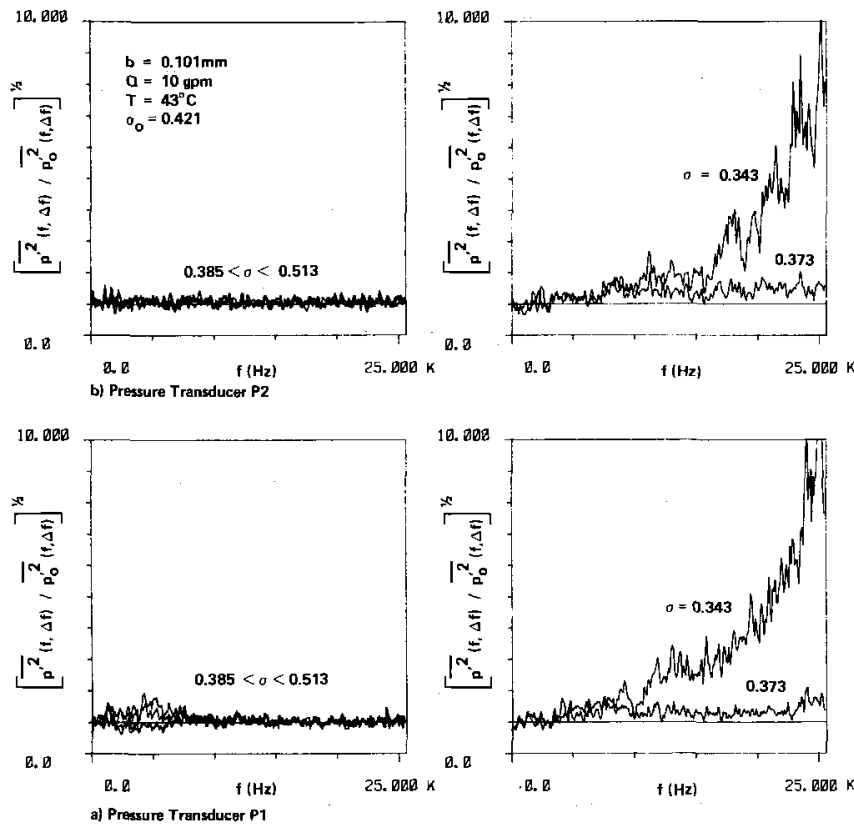

Fig. 19 Ratio of cavitating to noncavitating $\left(\sigma_{0}=0.421\right)$ spectral density for model valve at a fixed opening $\left(1 \mathrm{gpm}=6.31 \times 10^{-5} \mathrm{~m}^{3} / \mathrm{s}\right)$

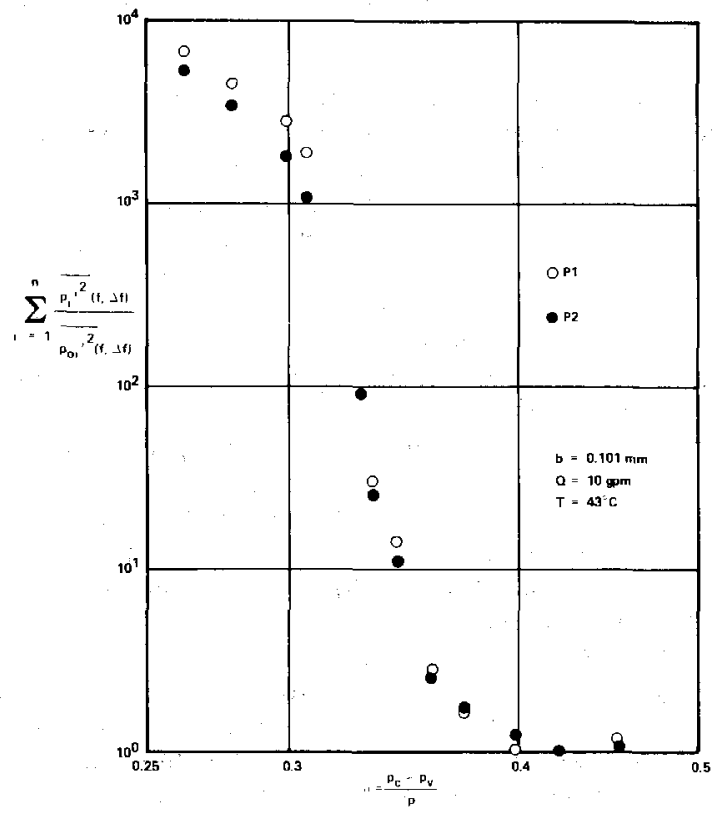

Fig. 20 Area under ratio of cavitating to noncavitating mean-square spectral density curve versus cavitation index for model valve at a fixed opening $\left(1 \mathrm{gpm}=6.31 \times 10^{-5} \mathrm{~m}^{3} / \mathrm{s}\right)$

larger opening and corresponding greater flow rate for the data of Fig. 21, the area function for $\boldsymbol{P}_{1}$ also varied by nearly four orders of magnitude from $\sigma=0.6$ to $\sigma=0.4$, while for $P_{2}$ the increase was not as great because of the hydrodynamic jet noise caused by the jet being directed toward that transducer. Nevertheless, the area functions of Figs. 20 and 21 are very sensitive indicators of cavitation events.

For both valves, it was found that, under identical conditions of valve opening $b$, flow rate $Q$, and temperature, the plots of energy with flow rate were repeatable. Comparisons of repeatability from test to test may be found in Martin, et al. [14]. Similarities in noncavitating spectra for the two valves are apparent in comparing the prototype valve results, Fig. 6, with those of transducer $P_{2}$ in the model, Fig. $12(b)$. As shown by Figs. 15(a) and $18(b)$, and more extensively in [14], spectra near cavitation inception are also qualitatively similar. 


\section{Cavitation Inception}

Attempts were made to formulate a criterion for the definition of incipient cavitation. Although the model was made of transparent walls for the purpose of visualization of the formation and collapse of bubbles, and the bubbles could be clearly seen as the valve was made to cavitate somewhat extensively, the onset of cavitation was extremely difficult to define visually because of the small openings, high jet velocities, and relatively small bubbles. After a number of somewhat unsuccessful attempts to establish incipient and desinent cavitation visually, reliance was placed upon the pressure transducers and the accelerometer, as well as aural sensing using a stethoscope. The identification of inception was much easier in the model using transducer $\boldsymbol{P}_{1}$ because of the dramatic change once cavitation commences. Because of the difficulty of separating jet noise from cavitation noise, the criterion for inception used for the prototype valve differed slightly from that applied to the model valve, the latter of which will be discussed first.

Model Valve. Because of the relatively low levels of energy in the model under noncavitating conditions, except when the jet was apparently directed onto transducer $P_{2}$, it was not difficult to sense the occurrence of a few cavitation events by comparing cavitating with noncavitating spectra. The data were taken by maintaining a constant flow with the spool locked in a fixed position. The cavitation number was reduced as the pressure difference $\Delta p$, and consequently the discharge $Q$, was maintained constant as nearly as possible. For each value of $\sigma$, the dynamic signal for transducer $P_{1}$ was processed and the total fluctuating energy noted. It was observed that this value hardly changed under noncavitating conditions. Cavitation inception was defined at the condition for which this nominal value increased by approximately 50 to 100 percent; which corresponded to only the slightest change in the cavitation index. Usually aural sensing by means of a stethoscope yielded the same value of the critical cavitation number. The cavitation number at the 50 to 100 percent energy increase is defined as the incipient cavitation index $\sigma_{i}$, and is plotted as a function of the Reynolds number in Fig. 22. The two sets of data correspond to a range of flow rates for each value of the fixed opening $b$.

Prototype Valve. As mentioned earlier, a comparison of fluctuating energies measured by the pressure transducer in the chamber of the prototype valve did not clearly indicate the difference between cavitation inception or limited cavitation and no cavitation because of the high level of jet noise. The points shown on Fig. 14 for $\sigma>0.6$ illustrate the difficulty inherent with comparing solely mean-square energy, while the spectra on Fig. 15 suggest there is no cavitation. Only by comparing spectra or by normalizing the spectral density with the corresponding noncavitating spectra, Fig. 16, could the presence of cavitation be determined. The criterion for incipient cavitation for the prototype valve was defined as the departure of the area under the normalized pressure spectral density curves versus cavitation number from the noncavitating value, which usually varied from approximately 0.9 to 1.1 , depending upon which noncavitating test was chosen as the reference value. The value of $\sigma_{i}$ was obtained from expanded versions of Fig. 17 by interpolating between the intersection of the extrapolated curve on the cavitating leg of the plot and the noncavitating horizontal line. Various criteria yielded differences in the incipient cavitation number of only 0.005 to 0.01 . The results of two series of tests at virtually the identical valve opening and nearly the same range of flow rates $4.42 \times 10^{-4}$ to $8.20 \times 10^{-4} \mathrm{~m}^{3} / \mathrm{s}(7$ to $13 \mathrm{gpm})$ are shown in Fig. 22. The only difference in the two series was the oil temperature, which resulted in a significant difference in the range of Reynolds numbers.

Although the use of the area function was not employed for

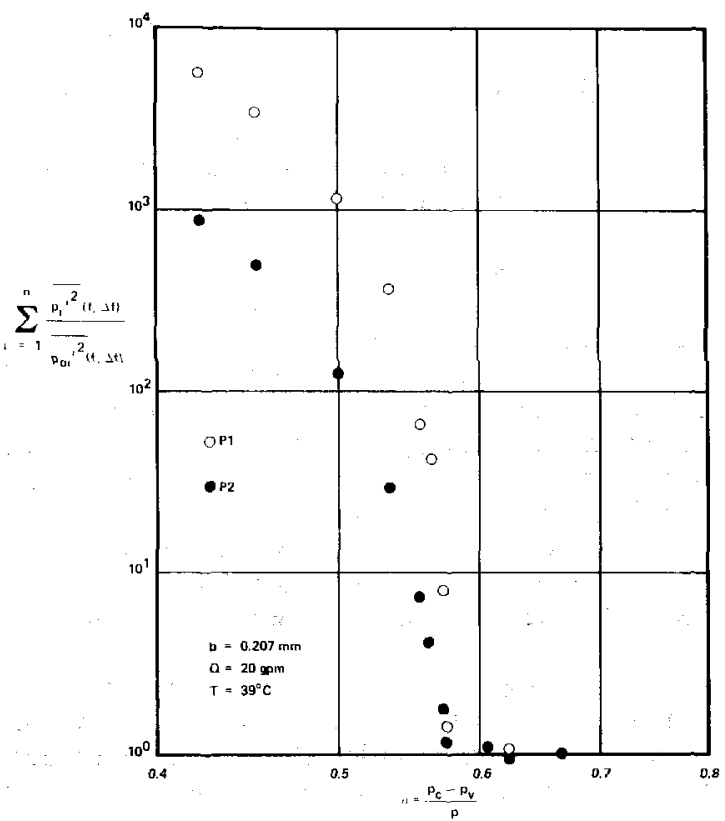

Fig. 21 Area under ratio of cavitating to noncavitating mean-square spectral density curve versus çavitzation index for model valve at a fixed opening $\left(1 \mathrm{gpm}=6.31 \times 10^{-5} \mathrm{~m}^{3} / \mathrm{s}\right)$

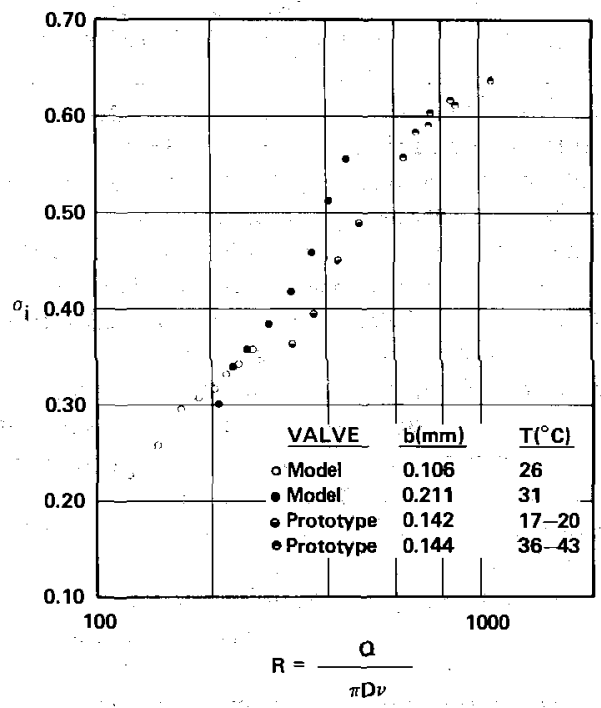

Fig. 22 Incipient cavitation index versus Reynolds number for model and prototype valves

the model valve, inspection of Figs. 20 and 21 illustrates that such a criterion could be as valid, or even more so, as it was for the prototype valve. The expanded $\sigma$ scale on Fig. 20 suggests that the effect of an increasing number of cavitation events as $\sigma$ is lowered below $\sigma_{i}$, which is somewhat of an arbitrary criterion, is to produce an exponential effect on the area function. The use of an energy spectrum with 50 ensemble averages does yield a reasonable statistical summary of cavitation events, however.

\section{Discussion of Results}

The criteria of cavitation inception employed in this investigation corresponds to a few, but unknown number, of cavitation events during the 50 ensemble averages taken over the sampling period. Single bubble formation in the transparent model could not be observed under these conditions. In fact, the cavitation index corresponding to the formation of a series of bubbles around the annulus was approximately 0.18 
less than the values shown on Fig. 22. Even a desinent cavitation number defined at the condition of the elimination of all visible bubbles was 0.10 smaller than the corresponding values of $\sigma_{i}$ depicted on Fig. 22. Because of the smallness of the bubbles, the high jet velocity, and apparently few cavitation events, bubbles at the condition $\sigma_{i}$ are difficult to observe.

No attempt was made to measure the size of free nuclei in the oil, although the high pressure filter limited the absolute size of any contaminant to $3 \mu \mathrm{m}$. Because of the continual filtering of the test liquid, the nuclei size and distribution probably did not vary much from test to test, as was evident from a comparison of energy spectra from test series to test series at the same conditions. As shown by the data on Fig. 6 , for two different temperatures there is an apparent viscous effect on the noncavitating energy spectra. Since cavitation inception is affected by the level of turbulence, as shown by Arndt $[32,33]$, the variation of $\sigma_{i}$ with Reynolds number shown in Fig. 22 can be directly correlated with the noncavitating fluctuating energy shown in Fig. 6. The data of McCloy and Beck [3] indicate a similar trend of $\sigma_{i}$ versus $R$ for two two-dimensional models. Their incipient cavitation index was based on the aural detection of the initial presence of a sharp crackling sound. For the range of Reynolds numbers, the critical values of $\sigma_{i}$ reported by McCloy and Beck [3] are 0.05 to 0.10 lower than the corresponding ones plotted on Fig. 22, however. Perhaps the criterion employed in this investigation corresponds to a smaller number of cavitation events at $\sigma_{i}$. The effect of viscosity on cavitation inception is also evident in the results of Backé and Riedel [5] and Riedel [6], who evidently defined inception at the condition of the change in the discharge coefficient of an orifice, or a situation correspondiong to wider spread cavitation. Noise data taken by Rouse [34] with a water jet issuing from a nozzle yielded $\sigma_{i}=0.55$ from a plot of noise versus $\sigma$. Intermittent bursts of noise were observed for values of $\sigma$ as high as $\mathbf{0 . 7}$, however, Obviously, the criterion employed for cavitation inception is somewhat subjective.

For the range of values of dissolved air content present in this study, there was no apparent effect of hysteresis on $\sigma_{i}$. The critical $\sigma_{i}$ did not appear to depend upon whether the downstream pressure was being decreased or increased. As discussed later, for dissolved air content at the two extremes experienced in the investigation -4.5 and 9.0 percent by volume - there was no apparent difference in $\sigma_{i}$ nor cavitation noise over the entire range of the cavitation index. Evidently the low residence time of the bubbles in the jet and valve chamber precluded any significant effect of gaseous cavitation. Even for the lowest values of the cavitation number experienced by the model valve, the bubbles disappeared as the flow left the downstream port, suggesting the presence of only vaporous cavitation. Baker, et al. [35], also found in their study of cavitation inception in confined jets that $\sigma_{i}$ was independent of gas content if the liquid was undersaturated at test-section pressure.

Although the accelerometer mounted on the end of the spool of the prototype valve was not quite as sensitive to the onset of cavitation, it proved to be quite a good indicator of the level of cavitation once inception occurred. As the cavitation index was lowered toward $\sigma_{i}$, as represented by Fig. $13(b)$, there was a very gradual increase in the fluctuating acceleration levels, making the formulation of a criterion of cavitation inception on the basis of the accelerometer quite difficult. The low level response on the accelerometer for $\sigma>$ $\sigma_{i}$ is attributed to mechanical effects caused by the method utilized to vary $\sigma$ - the operation of downstream valves.

\section{Conclusions}

Changes in flow pattern and jet orientation in the chamber of spool valves can be sensed by high-frequency response pressure transducers strategically placed. Under noncavitating conditions, jet noise can frequently be directly correlated with the valve opening and flow rate. For the Reynolds numbers tested in this study, there is a definite effect of viscosity on pressure fluctuations emanating from a turbulent noncavitating jet.

The effect of dissolved gas content on cavitation in spool valves is minimal if the gas content does not exceed that corresponding to atmospheric conditions.

High-frequency response pressure transducers are good diagnostic tools for detection of cavitation inception and cavitation intensity, while a high-frequency response accelerometer is a sensitive indicator of noise levels under cavitating conditions.

The ratio of cavitating noise to noncavitating noise is greater at higher frequencies than at lower frequencies, especially if there is the direct influence of jet noise.

Cavitation inception can be identified most easily by $(a)$ comparison of auto power spectra and $(b)$ ratio of cavitating spectra to noncavitating spectra for the same test conditions. In the case of a high-energy confined jet, root-mean-square values can be much less valid in indicating cavitation inception than individual spectra and, in fact, can be misleading.

For the range of data presented in this paper, the critical cavitation number is a function of the Reynolds number.

\section{Acknowledgments}

This investigation was supported in full by the Air Force Aero Propulsion Laboratory through AFOSR Contract F33615-77-C-2036, which was administered by Project Engineer Paul D. Lindquist. The authors would also like to acknowledge the direct contribution of J. I. Craig, F. D. Lewis, and H. J. Bates.

\section{References}

1 McCloy, D., and Martin, H. R., The Control of Fluid Power, Longman, London, 1973.

2 MacLellan, M. A., Mitchell, A. E., and Turnbull, D. E., "Flow Characteristics of Piston-Type Control Valves," Proceedings of the Symposium on Recent Mechanical Engineering Developments in Automatic Control, Institution Mechanical Engineers, London, Jan. 1960, pp. 13-30.

3 McCloy, D., and Beck, A., "Some Cavitation Effects in Spool Valve Orifices," Proceedings Institution Mechanical Engineers, Vol. 182, Part 1, No. 8, 1967-1968, pp. 163-174.

4 Backé, W., and Benning, P., "Über Kavitationserscheinungen in Querschnittsverengungen von ölhydraulischen Systemen," Industrie-Anzeiger, Vol. 63, 1962, pp. 35-42.

5 Backé, W., and Riedel, H.-P., "Kavitation in ölhydraulischen Systemen,"'Industrie-Anzeiger, Vol. 94, 1972, pp. 153-158.

6 Riedel, H.-P., "Kavitationsverhalten von verschiedenen Druckflüssigkeiten," Industrie-Anzeiger, Vol. 94, 1972, pp. 1724-1727.

7 Kleinbreuer, W., "Kavitationserosion in hydraulischen Systemen," Industrie-Anzeiger, Vol. 99, 1977, pp. 609-613.

8 Kleinbreuer, W., "Untersuchung der Werkstoffzerstörung durch Kavitation in ölhydraulischen Systemen," Dr.-1ng. dissertation, Technical University of Aachen, $1979,306 \mathrm{pp}$.

9 Eich, O., "Massnahmen zur Minderung von Kavitationsgeräuschen in Geräten in Ölhydraı!lik,"'Industrie-Anzeiger, Vol. 98, May 1976, pp. 739-743.

10 Eich, O., "Entwicklung geräuscharmer Ventile der Ölhydraulik;"' Dr.Ing. dissertation, Technical University of Aachen, 1979, $157 \mathrm{pp}$.

11 Lichtarowicz, A., and Pearce, I.D., "Cavitation and Aeration Effects in Long Orifices," Cavitation Conference, Institution Mechanical Engineers, Edinburgh, Sept. 3-5, 1974, pp. 129-144.

12 McCloy, D., and Beck, A., "Flow Hysteresis in Spool Valves," Proceedings of the First BHRA Fluid Power Symposium, CranfieId, 1969, pp. $170-182$.

13 Wiggert, D. C., Martin, C. S., and Medlarz, H., "Cavitation Damage Mechanisms: Review of Literature,"' Technical Report AFAPL-TR-79-2125, U.S. Air Force Aero Propulsion Laboratory, Feb. 1980, 53 pp.

14 Martin, C. S., Wiggert, D. C., and Medlarz, H., "Cavitation Damage Mechanisms: Experimental Study of Cavitation in a Spool Valve," Technical Report AFAPL-TR-79-2121, U.S. Air Force Aero Propulsion Laboratory, Feb. $1980,131 \mathrm{pp}$. 
15 Robertson, J. M., Hydrodynamics in Theory and Application, PrenticeHall, New York, 1965

16 Albertson, M. L., Dai, Y. B., Jensen, R. A., and Rouse, H., "Diffusion of Submerged Jets," Transactions, ASCE, Vol. 115, 1950, pp. 639-664.

17 Gutmark, E., and Wygnanski, I., "The Planar Turbulent Jet," Journal of Fluid Mechanics, Vol, 73, 1976, pp. 465-495.

18 Reethof, G., "Turbulence-Generated Noise in Pipe Flow," Annual Review of Fluid Mechanics, Vol. 10, 1978, pp. 333-367.

19 Lush, P. A., "Measurements of Subsonic Jet Noise and Comparison with Theory," Journal of Fluid Mechanics, Vol. 46, 1971, pp. 477-500.

20 Arndt, R. E. A., "Cavitation in Fluid Machinery and Hydraulic Structures,"' Annual Review of Fluid Mechanics, Vol. 13, 1981, pp. 273-328.

21 George, W. K., "The Equilibrium Range of Turbulent Pressure Spectra," Bulletin, American Physical Society, Vol. 19, 1974, p. 1158.

22 Fuchs, H. V., and Michalke, A., "On Turbulence and Noise of an Axisymmetric Shear Flow,' Journal of Fluid Mechanics, Vol. 70, 1975, pp. 179-265.

23 George, W. K., and Beuther, P. D., "Pressure Spectra in a Turbulent Shear Flow," Bulletin, American Physical Society, Vol. 22, 1977, p. 1285.

24 Ross, D., Mechanics of Underwater Noise, Pergamon Press, New York, 1976.

25 Jorgensen, D. W., "Noise from Cavitating Submerged Water Jets," Journal of the Acoustical Society of America, Vol. 33, Oct. 1961, pp. 1334-1338.

26 Deeprose, W. M., King, N. W., McNulty, P. J., and Pearsall, 1. S., "Cavitation Noise, Flow Noise and Erosion," Cavitation Conference, In stitution Mechanical Engineers, Sept. 3-5, 1974, Edinburgh, pp. 373-381.

27 Öba, R., and Itō, Y., "Cavitation Shock Pressures in a Venturi," ASME Cavitation and Polyphase Flow Forum, 1975, pp. 2-6.

28 Blake, W. K., Wolpert, M. J., and Geib, F. E., 'Cavitation Noise and Inception as Influenced by Boundary-Layer Development on a Hydrofoil," Journal of Fluid Mechanics, Vol. 80, 1977, pp. 617-640.

29 Tullis, J. P., and Govindarajan, R., "Cavitation and Size Scale Effects for Orifices," Journal of the Hydraulics Division, ASCE, Vol, 99, Mar. 1973, pp. $417-430$

30 Ball, J. W., and Tullis, J. P., "Cavitation in Butterfly Valves," Journal of the Hydraulics Division, ASCE, Vol. 99, Sept. 1973, pp. 1303-1318.

31 Tullis, J. P., "Testing Valves for Cavitation," Cavitation Conference, Institution Mechanical Engineers, Sept. 3-5, 1974, Edinburgh, pp. 47-55.

32 Arndt, R. E. A., "Pressure Fields and Cavitation," Proceedings of the 7th IAHR Symposium on Hydraulic Machinery, Vienna, 1974, Paper IX.

33 Arndt, R. E. A., "Investigation of the Effects of Dissolved Gas and Free Nuclei on Cavitation and Noise in the Wake of a Sharp Edged Disk," Proceedings of Joint ASME-IAHR-ASCE Symposium on Hydraulic Machinery, Fort Collins, Colo., 1978, pp. 543-556.

34 Rouse, H., "Cavitation in the Mixing Zone of a Submerged Jet," $L a$ Houille Blanche, Vol. 8, No. 1, 1953, pp. 9-19.

35 Baker, C. B., Holl, J. W., and Arndt, R. E. A., "The Influence of Gas Content and Polyethylene Oxide Additive upon Confined Jet Cavitation in Water," ASME Cavitation and Polyphase Flow Forum, 1976, pp. 6-8.

\section{I S C USS I O N}

\section{R. G. Cunningham}

The authors correctly note that cavitation information for oil hydraulic systems is relatively scarce. Their wellinstrumented study of two spool valves is a valuable contribution particularly in terms of detecting cavitation using mean-square energy measurements vis-a-vis energy spectra and more commonly used methods such as observation of bubbles and aural detection of the onset of cavitation.

Cavitation is a problem because of resulting noise, or damage, or both. From a design standpoint, cavitation is important if it is severe enough to cause a departure of performance from the theoretical model. Low-intensity cavitation, i.e., below these thresholds is of research interest but not from an engineering design point of view.

A prime objective is the establishment of a cavitation coefficient which predicts the onset of cavitation and which is relatively unaffected by the internal flow contours of the fluid machinery.

It would appear that the authors' equation defining the cavitation coefficient $\sigma$ in equation 6 could be improved. The

\footnotetext{
${ }^{1}$ The Pennsylvania State University, University Park, Pa. 16802.
}

denominator $\Delta p=\left(p_{L}-p_{R}\right)$ is a poor approximation to the dynamic or jet pressure $\rho V_{c}^{2} / 2$; while the use of $p_{R}$ is convenient from a pressure measurement standpoint, it is seriously flawed because the pressure rises from $p_{c}$ to $p_{R}$, essentially by a "sudden expansion" deceleration and disorganized flow pattern which may include reentry flow. The use of $\Delta p=\left(p_{L}-p_{c}\right)$ seems more logical as an index to dynamic pressure and also less sensitive to hardware design effects.

Caution should be exercised regarding the apparent immunity of this cavitation to the dissolved air-content of the hydraulic oil. Cavitation in oil systems-while producing the same undesirable effects as water cavitation-differs in two significant respects, (1) the vapor pressure of an oil is relatively low and complicated by the fact that an oil is a mixture of components ranging from light to heavy ends. (2) The solubilities of gases are much larger in organic liquids such as oils, than in water. The solubility of air in water (room temperature and pressure) is about 2 percent by volume; it ranges from 8-10 percent for lubricating oils, 12 percent for diesel fuel and 18-25 percent for gasoline. As a result, "gas cavitation" occurs readily in oil systems if the oil is at or near saturation conditions [1].

The authors' test conditions produced oil with an extremely low air content. The solubility of air in oil follows Henry's law, i.e., the mass of gas dissolved is proportional to the partial pressure of the gas in the space above the liquid under equilibrium conditions. The extremes of dissolved air reported (4.5 percent up to 9 percent at 14.7 psia) translate to only 0.013 percent and $\mathbf{0 . 0 2 6}$ percent for this oil raised to the pressure of 5000 psia implied for some of the prototype tests. Air nuclei are important as the sites of cavitation events. At least in static bodies of liquid, extreme deaeration delays or prevents vapor cavitation upon pressure release. Flowing liquids at some extreme degree of deaeration may similarly become resistant to vapor cavitation-and perhaps also more sensitive to the vapor pressure of the oil.

Transient or hysteresis cavitation effects have been noted in other oil systems [2, 3] containing dissolved air. Air evolves quite rapidly on release of pressure-e.g., in a venturi throat-and then dissolves again relatively slowly, upon pressurization. As a result some oil systems with large pressure gradients effectively deaerate the fluid during warmup. This subject of this work-high-speed actuators and spool valves-may well be affected by this phenomenon.

\section{References}

1 Schweitzer, P. H., and Szebehely, V. G., "Gas Evolution in Liquids and Cavitation,' J. of Applied Physics, Vol. 21, December 1950, pp. 1218-1224.

2 Cunningham, R. G., and Brown, F. B., "Oil Jet Pump Cavitation," ASME 1970 Cavitation Forum, May 24-27, 1970, Detroit Michigan, pp. 13-16.

3 Cunningham, R. G., "The Jet Pump as a Lubrication Oil Scavenge Pump for Aircraft Engines," The Pennsylvania State University, WADC Tech. Rept., July 1954, pp. 55-143.

\section{A. Lichtarowicz ${ }^{2}$}

The authors should be congratulated on their interesting paper describing methods of detecting cavitation inception in spool valves. As pointed out in the paper, it is very dificult to establish the exact point of cavitation inception in an actual piece of engineering hardware.

The comparison of the ratio of cavitating to noncavitating energy spectra, as used by the authors, seems to be a very useful method of detecting cavitation inception, though perhaps it is a little cumbersome.

The writer, from his experiences, fully endorses the 


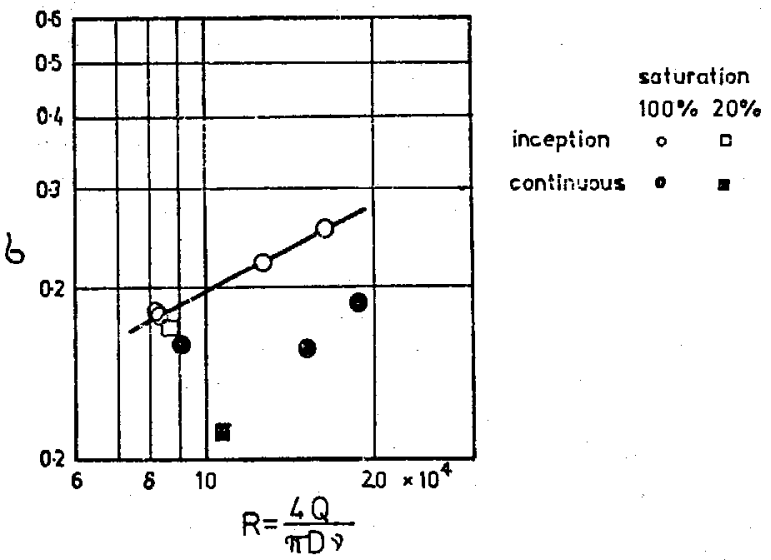

Fig. 1 Cavitation index-Reynolds number variation for shape edge orifice plate

statement made that in laboratory models the use stethoscope gives a very reliable indication of the inception point which agrees with the data obtained with much more sophisticated electronic equipment.

Another useful method used by the writer [reference A] is to place a small "hydrophone" made from a piezo-electric crystal in the vicinity of the emerging jet, but not in its path. The change in the output as observed on an oscilloscope screen proved to be very reliable method of detecting the cavitation inception, especially as cavitation initially occurred in bursts.

Figure 1 shows results extracted from Pearce [B]. They relate to the cavitation inception for a sharp edge orifice plate (similar to that used for flow measurement). The orifice diameter was $2.5 \mathrm{~mm}$ and the test fluid was aviation kerosene. Each point was obtained by holding the downstream pressure constant and increasing the upstream pressure till the cavitation was well established. The results are similar to the author's Fig. 22. Two additional points were obtained with reduced air content in the test liquid. These points seem to confirm the author's statement that the air content changes do not affect the cavitation inception, at least for the range of air contents tested.

Finally the writer would like to sound a word of warning. Both the authors' Fig. 22 and Fig. 1 in the discussion relate the cavitation number with Reynolds number. In both cases the variation Reynolds number was obtained mainly by flow variation and by size variation, but fluid properties varied only by a factor of about 2 . To ensure that these graphs can be generalized, further testing with different liquids is required.

\section{Additional References}

A. Lichtarowicz, A., and Pearce, I. D. "Cavitation and acration effects in Iong orifices," Conf. on Cavitation, Institution of Mechanical Engineers, London, 3-5 Sept 1974.

B. Pearce, I. D., "Cavitation in Orifices," Ph.D. thesis, University of Nottingham. July 1969.

\section{Author's Closure}

The comments of Dr. Cunningham are most welcome as they address issues that were not discussed in detail in the paper. Except for the consideration of noise he is correct in stating that the low-intensity cavitation very close to inception is of only limited consequence in oil hydraulic systems.

The definition of the pressure difference across the value $\Delta p=p_{L^{-}}-P_{R}$ was chosen rather than $p_{L}-p_{c}$ because of the influence of partial or complete jet stagnation of $p_{c}$. For all of the data, the difference in $\Delta p$ for the two definitions was less than 4 percent. For the prototype valve the chamber pressure was larger than $p_{R}$, while for the model valve the opposite was observed. In either case the cavitation index would not have differed by more than 4 percent.

The authors recognize that air is much more soluble in hydraulic oil than in water and the attendant likelihood of enhanced gaseous cavitation. Although the local instantaneous pressures in the turbulent jet surely dropped to the saturation pressure, which was atmospheric or lower, the mean pressure in each valve chamber was always well above atmospheric pressure at cavitation inception. It should also be noted that the residence time for the fluid in each valve was small, inhibiting any significant growth of air bubbles by gaseous diffusion.

The maximum upstream pressure $p_{L}$ was 2700 psi and 400 psi for the prototype and model valve, respectively. The 500 psi pressure quoted in the paper only related to the maximum level attainable by the hydraulic pump. Most hydraulic systems are not saturated with air at the high pressures mentioned by Dr. Cunningham. 